\title{
Dimensionally Adaptive $h p$-Finite Element Simulation and Inversion of 2D Magnetotelluric Measurements
}

\author{
J. Alvarez-Aramberri ${ }^{\mathrm{a}, *}$, D. Pardo ${ }^{\mathrm{b}, \mathrm{a}, \mathrm{c}}$ \\ ${ }^{a}$ Basque Center for Applied Mathematics (BCAM), Mazarredo 14, 48009, Bilbao, Spain \\ ${ }^{b}$ University of the Basque Country (UPV/EHU), Bilbao, Spain \\ ${ }^{c} I K E R B A S Q U E$, Basque Foundation for Science, Bilbao, Spain
}

\begin{abstract}
Magnetotelluric (MT) problems often contain different subdomains where the conductivity of the media depends upon one, two, or three spatial variables. Traditionally, when a MT problem incorporates a three-dimensional (3D) subdomain, the numerical method employed for simulation and inversion was 3D over then entire domain. In here, we propose to take advantage of the possibly lower dimensionality of certain subdomains during the inversion process. By doing so, we obtain significant computational savings (up to $75 \%$ in some scenarios) and increased accuracy on the results. We numerically illustrate this method by employing two dimensional (2D) computations based on a multi-goal oriented $h p$-adaptive Finite Element Method (FEM) that exhibits superior convergence properties. Additionally, we provide a formulation for implementing an efficient adjoint based method for the computation of the derivatives of the impedance, and we show the importance of the (a) proper selection of the inversion variable, and (b) the advantages of using both the Transverse Electric (TE) and Transverse Magnetic (TM) measurements for the proper inversion of MT data.
\end{abstract}

Keywords: Dimensionally Adaptive Method, Magnetotelluric Problem, Finite Element Method (FEM), $h p$-adaptivity, Inverse Problems.

\footnotetext{
*Corresponding author

Email address: julen.alvarez.aramberri@gmail.com (J. Alvarez-Aramberri)
} 


\section{Introduction}

In the decade of the 50's, Tikhonov [1] and Cagniard [2] established the theoretical foundations of the magnetotelluric (MT) method, an Earth exploration technique based on electromagnetic (EM) measurements governed by Maxwell's equations, intended to provide an image of the Earth's subsurface. In this method, several receivers are placed a few centimeters below the Earth's surface, and in contrast to other measurement acquisition systems such as control source EM or borehole logging instruments, it employs a non-artificial (natural) source located at the ionosphere. The MT method is a noninvasive technique with a negligible environmental impact that is capable of studying distances ranging from between tens of meters to hundreds of kilometers. Due to this, the MT method has generated great interest over the last years. It has been employed, inter alia, in $\mathrm{CO}_{2}$ geological storage [3], crustal exploration [4], groundwater monitoring [5], and earthquake precursor prediction research $[6,7]$.

The proper interpretation of MT measurements needs from accurate numerical simulations. Among all possible methods suitable for simulating MT measurements, we focus on higher order methods, which are able to achieve a remarkable level of accuracy. These methods have been extensively employed in the last years in applied mathematics to solve a variety of engineering problems. For example, the isogeometric analysis (IGA) [8, 9] has recently experimented a huge explosion and it has been widely applied to the engineering industry, as well as the more recent Discontinuous Petrov-Galerkin (DPG) method initially proposed by Demkowicz and Gopalakrishnan $[10,11]$, or the self-adaptive $h p$-Finite Element Method (FEM) $[12,13]$ (where $h$ stands for the element size and $p$ for the polynomial order of approximation associated to each element). The latter one has been recently employed, for instance, to model the bone conduction of sound in the human head [14], or to simulate bend, step, and magic-T electromagnetic waveguide structures [15].

In this work, we develop an extension of the self-adaptive $h p$-FEM originally proposed by Demkowicz et al. $[12,13]$. The $h p$-FEM allows for both $h$ and $p$ local refinements across elements, which constitutes a notorious advantage, since the combination of both types of refinements enables to better capture the presence of singularities, thereby providing low discretization errors. It is thus a highly accurate method that provides exponential convergence rates $[16,17]$ even when these singularities are present, a typical situation that occurs in MT. 
In the MT forward problem, accurate results are only necessary at the receivers. Hence, the goal-oriented adaptivity (first proposed by Becker and Rannacher in the mid 90's [18]) becomes a natural choice to build the finite element mesh. The objective of traditional goal-oriented strategies is to construct an optimal grid in the sense that it minimizes the problem size needed to achieve a preselected tolerance error in a given physical magnitude of engineering interest (in our case, a component of the EM fields). Since in MT it is necessary to obtain accurate results at multiple receiver positions, in here we employ a multi-goal oriented self-adaptive algorithm originally proposed by Pardo in [19]. One of the main advantages of this approach is that we are able to measure the error in a geophysically meaningful norm.

Our initial study on the use of $h p$-FEM for MT was developed on the conference paper [20], where we extended the multi-goal oriented adaptive $h p$ FEM $[12,19]$ to the case of simulation of 2D MT problems. After that, in [21] we designed an automatically adapted Perfectly Matched Layer (PML) [22, $23]$ suitable for scenarios where the material properties change abruptly, as the case of air-ground interface in MT. In a subsequent conference paper [24], we noticed that MT quantities of interest (QoI), namely impedances, exhibit a superior convergence behavior than that offered by the EM fields themselves. In [23], we developed a secondary field formulation where the primary field was given by an exact one dimensional (1D) solution [25] (rather than the traditional solution over a homogeneous space) in order to reduce the computational cost of solving a direct problem without reducing the accuracy.

Motivated by the aforementioned previous work, in this paper we further exploit the idea of dimensional adaptivity based on lower dimensional primary fields. Given a forward simulation, it is often possible to decompose the problem according to its dimensionality by considering different dimensions for different regions. Thus, a good dimensionality analysis enables to determine the zones of the domain that can be modeled with $1 \mathrm{D}$ or $2 \mathrm{D}$ discretizations. Based on this idea, we propose an inversion algorithm that takes advantage of a domain decomposition driven by the dimensionality of each subdomain. We first obtain a solution for the lowest dimensional inverse problem (IP). Then, we solve a higher dimensional IP by employing the information of lower dimensional IPs. This idea relies upon the results obtained in [26] and [27], where authors show that measurements often exhibit a dimensionality signature that can be properly exploited when solving IPs. 
Other contributions of this work show: (a) convergence results on MT problems illustrating the superior convergence properties of $h p$-FEM vs. $h$ and $p$-FEM, as predicted by the theory $[16,17],(\mathrm{b})$ a sequence of optimal $h p$-grids delivered by the $h p$-adaptive FEM, which in contrast to $h p$-grids obtained in [20] for the TE mode, exhibit heavy refinements in the proximity of the PML, as physically expected, (c) the impact of the selected variable for the inversion process, and (d) a joint interpretation of Transverse Electric (TE) + Transverse Magnetic (TM) data, which provides complementary information that enables to recover a more accurate map of the Earth's subsurface than with either TE or TM measurements alone, as shown, for instance, in [28], Chapter 4. Additionally, we also provide an efficient and computationally inexpensive approach based on the adjoint problem to compute the derivatives of the impedance (a nonlinear QoI) with respect to any variable used for the inversion.

For solving the nonlinear optimization problem dictated by the IP, we employ the L-BFGS-U (Broyden-Fletcher-Goldfarb-Shanno) method [29]. The computational domain of the forward problem is truncated with a Perfectly Matched Layer [23], and we assume an underlying 1D resistivity distribution of the subsurface where some 2D inhomogeneities are located. The application of the $h p$-FEM in MT is still on an early stage. Being the $h p$-FEM a very accurate method, our main concern before applying it to more complex MT scenarios is to study and guarantee its suitability for the simulation and inversion of MT measurements. This also motivates the use of an underlying 1D layered media, because it allows us, for instance, to compare the numerical solutions with an exact one. Nevertheless, this simple scenario illustrates the superior convergence properties of the $h p$-FEM and its suitability to solve the IPs in MT.

This work summarizes part of the main results shown on the first author's Ph.D. Dissertation [30]. Other than that, the work described in this paper has not been published in any other form, and it constitutes a significant advance with respect to previous publications. Specifically, (a) for the inversion, we employ the impedance and we consider both TE and TM modes, which were not considered on [20] and are essential for the proper interpretation of MT measurements, (b) we show the faster convergence of the $h p$-adaptivity in comparison with uniform $h$ and $p$ refinements for the MT problem, (c) for both modes we obtain optimal grids (only TE mode was considered in [23]), in a realistic scenario where we also include the air layer (that was not considered in [20]), and (d) we propose and illustrate for the 
first time in MT, the benefits of including the dimension of the problem as a variable in the inversion process.

The present work is organized as follows. In Section 2 we formulate the direct and inverse problems and we provide the discretization methods to solve them. In Section 3 we introduce the dimensionally adaptive method for the inversion, and we show the numerical results in Section 4. Finally, the conclusions are outlined in Section 5.

\section{Formulation}

\subsection{Direct Problem}

Assuming isotropic materials, Maxwell's equations can be written in frequency domain as:

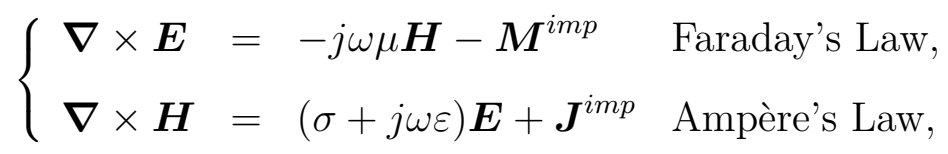

where $j$ is the imaginary unit, $\omega$ the angular frequency, $\mu$, and $\varepsilon$ stand for the magnetic permeability and electrical permittivity, respectively, and $\sigma$ for the conductivity $\left(\rho=\sigma^{-1}\right.$ is the resistivity) of the media. $\boldsymbol{E}=\left(E_{x}, E_{y}, E_{z}\right)$ and $\boldsymbol{H}=\left(H_{x}, H_{y}, H_{z}\right)$ are the electric and magnetic fields in the frequency domain, driven by an impressed prescribed electric and magnetic density current sources, defined by $\boldsymbol{J}^{i m p}=\left(0, J_{y}, 0\right)$ and $\boldsymbol{M}^{i m p}=\left(0, M_{y}, 0\right)$, respectively.

Two independent and uncoupled formulations are naturally derived from Maxwell's equations when the materials and source depend only upon the two spatial variables $(x, z)$, the so called TE and TM modes. The first one involves $\left(E_{y}, H_{x}, H_{z}\right)$ field components, while the latter one only considers $\left(H_{y}, E_{x}, E_{z}\right)$.

While the $y$ component of the electric and magnetic fields satisfy scalar equations, the remaining field components satisfy coupled vectorial equations (see [30]). The scalar equations are often much easier to solve numerically. Moreover, once they are computed, the rest of the fields can be easily obtained via postprocessing. We focus and solve then these scalar equations, which are given by:

$$
-\boldsymbol{\nabla} \cdot\left(\mu^{-1} \nabla E_{y}\right)-h_{T E}(\sigma) E_{y}=-j \omega J_{y}^{i m p}
$$


and

$$
-\nabla \cdot\left(h_{T M}(\sigma) \nabla H_{y}\right)+j \omega \mu H_{y}=-M_{y}^{i m p},
$$

where $h_{T E}(\sigma)=\omega^{2} \varepsilon-j \omega \sigma$ and $h_{T M}(\sigma)=\frac{1}{\sigma+j \omega \varepsilon}$.

The impedance and the apparent resistivity, two employed quantities in MTs, are defined as:

$$
\mathcal{Z}_{l k}=\frac{E_{l}}{H_{k}} \quad \text { and } \quad \rho_{l k}^{a p p}=\frac{1}{\sigma_{l k}^{a p p}}=\frac{1}{\omega \mu}\left|\mathcal{Z}_{l k}\right|^{2},
$$

where $l$ and $k$ can be either $x$ or $y$.

The MT method is typically employed in regions with some prior geological knowledge of the media. This enables in practice to align the coordinate system with the medium strike direction. Thus, since the electric and magnetic fields are related only through their orthogonal fields (the diagonal components are zero because they are related to the parallel electric and magnetic fields), only $\mathcal{Z}_{y x}=\mathcal{Z}_{T E}$ and $\mathcal{Z}_{x y}=\mathcal{Z}_{T M}$ are different from zero.

Variational Formulations. We derive suitable variational formulations for the 2D MT forward problems for the two modes. To do so, we pre-multiply equations (2) and (3) by the complex conjugate of any scalar function that belongs to $V(\Omega)$, we integrate by parts, and we incorporate the homogeneous Dirichlet boundary conditions imposed in this work in the outer-part of the PML. We have that:

$$
\underbrace{\left\langle\nabla F, \mu^{-1} \nabla E_{y}\right\rangle_{L^{2}(\Omega)}-\left\langle F, h_{T E}(\sigma) E_{y}\right\rangle_{L^{2}(\Omega)}}_{b^{T E}\left(F, E_{y}\right)}=\underbrace{-j \omega\left\langle F, J_{y}^{i m p}\right\rangle_{L^{2}(\Omega)}}_{G^{T E}(F)},
$$

and

$$
\underbrace{\left\langle\nabla F, h_{T M}(\sigma) \nabla H_{y}\right\rangle_{L^{2}(\Omega)}+j \omega\left\langle F, \mu H_{y}\right\rangle_{L^{2}(\Omega)}}_{b^{T M}\left(F, H_{y}\right)}=\underbrace{-\left\langle F, M_{y}^{i m p}\right\rangle_{L^{2}(\Omega)}}_{G^{T M}(F)} .
$$

For the secondary field formulation, we denote the conductivity of the reference model (a 1D layered media in our case) as $\sigma^{P}$. Let $\Omega_{S} \in \Omega$ be the domain where the $2 \mathrm{D}$ inhomogeneities are located. Hence, defining $\sigma^{S}=\sigma-\sigma^{P}$, $\sigma^{S}$ is zero outside $\Omega_{S}$. We now split the EM fields into their primary and secondary contributions, $E=E^{P}+E^{S}, H=H^{P}+H^{S}$, and we define $h_{T M}^{S}(\sigma)=h_{T M}(\sigma)-h_{T M}^{P}(\sigma)$. 
Then, the sesquilinear forms for the secondary field formulation are identical, only changing the total fields expression by the secondary fields, while the right hand sides for the TE and TM modes are now given by $-j \omega\left\langle F, \sigma^{S} E_{y}^{P}\right\rangle_{L^{2}(\Omega)}$ and $-\left\langle\nabla F, h_{T M}^{S}(\sigma) \nabla H_{y}^{P}\right\rangle_{L^{2}(\Omega)}$, respectively.

These above expressions are well defined for $E_{y}, E_{y}^{P}, E_{y}^{S}, H_{y}, H_{y}^{P}, H_{y}^{S}$, and $F$ in $V(\Omega)=H_{\Gamma_{D}}^{1}(\Omega)=\left\{F \in L^{2}(\Omega):\left.F\right|_{\Gamma_{D}}=0, \nabla F \in \boldsymbol{L}^{2}(\Omega)\right\}$. From here, we define the problem to be solved with the following abstract variational formulation for the direct problem (D) and its associated adjoint problem (A) as:

$$
\begin{array}{llll}
D: & \text { Find } \hat{u} \in V(\Omega), \text { such that } & b(v, \hat{u})=G(v), & \forall v \in V(\Omega), \\
A: & \text { Find } \hat{v} \in V(\Omega), \text { such that } & b(\hat{v}, u)=P(u), & \forall u \in V(\Omega),
\end{array}
$$

where the hat denotes the solution to the problem. Thus, $\hat{u}$ can be either $E_{y}$ for the TE mode, or $H_{y}$ for the TM mode, $G(\cdot)$ and $P(u)$ are antilinear forms $\left(G^{T E}(\cdot)\right.$ stands for the TE mode, while $G^{T M}(\cdot)$ for the TM mode), and $b(\cdot, \cdot)$ is a sesquilinear form $\left(b^{T E}(\cdot, \cdot)\right.$ for the TE mode and $b^{T M}(\cdot, \cdot)$ for the TM mode) that we rewrite for convenience as:

$$
b(\cdot, \cdot):=b_{1}(\cdot, \cdot)+b_{2}(\cdot, \cdot ; h(\sigma)) .
$$

Here, $h$ is a function that depends on the considered mode, being $h=h_{T E}$ for the TE mode and $h=h_{T M}$ for the TM mode. Thus, $b_{1}$ is independent from $h$, while $b_{2}$ is not.

We define now the following linear Quantity of Interest (QoI) associated to the $i$-th receiver:

$$
L^{i}(u)=\frac{1}{\left|\Omega_{R^{i}}\right|} \int_{\Omega_{R^{i}}} u d \Omega .
$$

Here, $L^{i}(u)$ is a linear and continuous functional $[31,32]$ in $u$, which is associated with an scalar component of the EM fields solution at the $i$-th receiver (occupying a domain $\Omega_{R^{i}}$ ).

In the particular case in which $P(u)=L^{i}(u)$, then there is one adjoint problem for each of the $N_{\text {rec }}$ receivers. Since we use an LU factorization implemented by solver ("MUltifrontal Massively Parallel Solver") [33], the linear system of equations is factorized only once and the extra cost for solving each adjoint problem reduces only to backward and forward substitutions.

Discretization. We use the multi-goal oriented $h p$-FEM [19], an extension of the work of Demkowicz et al. $[12,13]$ to solve equations (7) and (8) accurately at all receivers simultaneously. 
To truncate the computational domain, we employ a PML, which maintains unchanged the continuous solution inside the region of interest. GomezRevuelto et al. [34] shows the suitability of using a PML in the context of $h p$-adaptive FEM, specially because it enables to match the high-accuracy level delivered by the $h p$-adaptive FEM. In particular, we use a PML [21] suitable for problems that involve high contrast properties between neighboring materials, which typically appear in MT problems on, for example, the air-ground interface. For additional details on the application of this PML to the MT problem, see [30].

\subsection{Inverse Problem}

- Given a discretization of the function that defines the model parameters

$$
m(\boldsymbol{x})=\sum_{i=1}^{M} m_{i}(\boldsymbol{x})
$$

where $m_{i}=m_{i}(\boldsymbol{x})=m^{i} \chi_{i}(\boldsymbol{x})$, with $m=\left\{m^{1}, m^{2}, \ldots, m^{M}\right\}$ being the $M$ degrees of freedom used to discretize the conductivity (or resistivity) distribution of the Earth's subsurface, and $\chi_{i}(\boldsymbol{x})$ being defined as:

$$
\chi_{i}(\boldsymbol{x})=\left\{\begin{array}{lll}
1 & \text { if } & x \in \Omega_{i}, \\
0 & \text { if } & x \notin \Omega_{i},
\end{array}\right.
$$

where $\Omega_{i}$ stands for a particular $i$-th rectangular block of the domain.

- The data $d=\left\{d^{1}, d^{2}, \ldots, d^{N}\right\}$ are $\mathrm{N}$ impedance measurements acquired at different surface locations and frequencies. Measurements have associated weights $\theta=\left\{\theta^{1}, \theta^{2}, \ldots, \theta^{N}\right\}$ to ponder its importance. Since we employ the impedance at several frequencies $\omega_{j}$, we divide the computed impedance by $\sqrt{\omega_{j}}$ to normalize their values.

We define the impedance-based cost functional (misfit data) as:

$$
C(m)=\sum_{k}^{N_{\text {mod }}} \sum_{\omega_{j}}^{N_{\omega}} \sum_{i}^{N_{\text {rec }}}\left|\frac{1}{\sqrt{\omega_{j}}}\left(\mathcal{Z}_{\omega_{j}}^{i, k}(m)-d_{\omega_{j}}^{i, k}\right)\right|^{2},
$$

where $N_{\text {mod }}$ corresponds to 1 if one single mode is employed for inversion (solving numerically equation (2) for the TE mode and equation (3) for the TM mode), or 2 if a joint inversion is performed, and therefore the two 
equations are solved. $N_{\omega}$ is the number of frequencies and $N_{\text {rec }}$ the number of receivers. In our numerical experiments, we consider no regularization term in order to compare exactly the same cost functional.

We deal then with a simple bounded nonlinear constrained optimization problem, where the objective is to seek argument $\hat{m}$ that minimizes cost functional $C(m)$ constrained to some simple (constant) bounds on $m$ that are imposed in order to avoid unphysical values of the variable (for example, to prevent negative values of conductivity).

To minimize $C(m)$, we employ a gradient based method based on a truncated Taylor's series expansion. Since the quality of such approximation depends upon the variable with respect to which we perform such expansion, the selection of the model parameters (inversion parameters) becomes crucial. It might be of interest then to perform the inversion with respect to a variable other than the conductivity. To do so, we study the convenience of considering the cost functional as a function of different model parameters, such as $m=\sigma, m=\rho$, or $m=\log (\rho)$.

$L-B F G S-U$ Method. We employ the L-BFGS-U method to solve the inverse problems. This gradient based method is suitable for simple bounded problems. It is implemented in FORTRAN [35], and it has been already employed in MT by Avdeev and Avdeeva [36]. Being a quasi-Newton method, in this particular case, the Hessian is replaced by a symmetric and positive definite matrix that is updated at every iteration according to the limited-memory BFGS formula [37, 38].

Computation of the Jacobian. The traditional theory of goal-oriented adaptivity employs a linear and continuous functional as QoI (see equation (10)). In the case of geophysical resistivity applications as MT, the appearance of nonlinear QoI as the impedance $\mathcal{Z}$ is common. Therefore, it is necessary to compute the derivatives of this nonlinear QoI with respect to the model parameters. Thus, in contrast to our previous work where only $E_{y}$ was considered as linear QoI [20], we now employ the impedance, a suitable nonlinear QoI. We define it in terms of the linear functionals obtained with the $h p$-FEM, and it is given at the $i$-th receiver by:

$$
\mathcal{Z}_{l k}^{i}=\frac{L^{i}\left(E_{l}\right)}{L^{i}\left(H_{k}\right)},
$$

where $l$ and $k$ can be either $x$ or $y$ in 2D Cartesian coordinates. 
We express now the analytical derivative of the impedance in terms of the derivatives of the linear functionals, which are computed numerically.

$$
\frac{\partial \mathcal{Z}_{l k}^{i}}{\partial m_{j}}=\frac{\frac{\partial L^{i}\left(E_{l}\right)}{\partial m_{j}} L^{i}\left(H_{k}\right)-\frac{\partial L^{i}\left(H_{k}\right)}{\partial m_{j}} L^{i}\left(E_{l}\right)}{\left(L^{i}\left(H_{k}\right)\right)^{2}}
$$

To estimate the entries of the first derivative of $L^{i}(u)$ with respect to each of the inversion variables $m_{j}$, we employ the chain rule:

$$
\left[\mathcal{J}_{L_{m}}\right]_{j}^{i}=\frac{\partial L^{i}(u)}{\partial m_{j}}=\underbrace{\frac{\partial L^{i}(u)}{\partial \sigma_{j}}}_{\left[\mathcal{J}_{L_{\sigma}}\right]_{j}^{i}} \frac{\partial \sigma_{j}}{\partial m_{j}},
$$

where $\mathcal{J}_{L_{m}}$ and $\mathcal{J}_{L_{\sigma}}$ are arrays of $N_{\text {rec }}$ vectors (one for each component of $L^{i}$ ) of dimension equal to the number of model parameters $M$. Notice that $\partial \sigma_{j} / \partial m_{j}$ is computed analytically, while $\mathcal{J}_{L_{\sigma}}$ numerically. A single numerical computation of the latter is then sufficient to obtain the derivative with respect to the selection of $m_{j}$.

To compute the entries of $\left[\mathcal{J}_{L_{\sigma}}\right]_{j}^{i}$, we employ the solutions of the forward direct and adjoint problems (equations (7) and (8), respectively) and we evoke the Representation Theorem [39], which states that:

$$
P(\hat{u})=G(\hat{v})=b(\hat{v}, \hat{u})
$$

Following [30], we have that:

$$
\left[\mathcal{J}_{L_{\sigma}}\right]_{j}^{i}=\frac{\partial L^{i}(\hat{u})}{\partial \sigma_{j}}=-b_{2}\left(\hat{v}^{i}, \hat{u} ; \frac{\partial h(\sigma)}{\partial \sigma_{j}}\right) .
$$

For the TE mode we define the following two linear operators associated to each receiver:

$$
L^{i}(u)=\frac{1}{\left|\Omega_{R^{i}}\right|} \int_{\Omega_{R^{i}}} u d \Omega, \quad \text { and } \quad L_{T E}^{i}(u)=\frac{1}{j \omega \mu\left|\Omega_{R^{i}}\right|} \int_{\Omega_{R^{i}}} \frac{\partial u}{\partial z} d \Omega .
$$

$L_{T E}^{i}(u)$ is defined according to the equation for $H_{x}$ given by Maxwell's equations. This EM field component is needed for computing the impedance. 
- If $P(u)=L^{i}(u)$ :

$$
\begin{array}{ll}
D: & b(v, \hat{u})=G(v), \quad \forall v \in V, \\
A: & b\left(\hat{v}_{E}^{i}, u\right)=L^{i}(u), \quad \forall u \in V .
\end{array}
$$

From (20) we obtain the solution for $\hat{E}_{y}=\hat{u}$, and we compute the associated linear QoI as $L^{i}\left(\hat{E}_{y}\right)$. By solving (21), we obtain the solution for $\hat{v}_{E}^{i}$. Since (20) stands for all $v$, in particular, it is also valid for $\hat{v}_{E}^{i}$. Then, using equation (17), we have that:

$$
\frac{\partial L^{i}\left(\hat{E}_{y}\right)}{\partial \sigma_{j}}=-b_{2}\left(\hat{v}_{E}^{i}, \hat{E}_{y} ; \frac{\partial h_{T E}(\sigma)}{\partial \sigma_{j}}\right)=\left\langle\hat{v}_{E}^{i}, \hat{E}_{y}\right\rangle_{L^{2}(\Omega)} .
$$

- If $P(u)=L_{T E}^{i}(u)$ :

$$
\begin{aligned}
& D: \quad b(v, \hat{u})=G(v), \quad \forall v \in V, \\
& A: \quad b\left(\hat{v}_{H}^{i}, u\right)=L_{T E}^{i}(u), \quad \forall u \in V .
\end{aligned}
$$

Since $\hat{E}_{y}$ is already computed, from Maxwell's equations we have that

$$
L^{i}\left(\hat{H}_{x}\right)=L_{T E}^{i}\left(\hat{E}_{y}\right) .
$$

Following the same argument, solving equation (24) we obtain the solution for $\hat{v}_{H}^{i}$, and using the Representation Theorem stated above, we obtain that:

$$
\frac{\partial L^{i}\left(\hat{H}_{x}\right)}{\partial \sigma_{j}}=\frac{\partial L_{T E}^{i}\left(\hat{E}_{y}\right)}{\partial \sigma_{j}}=-b_{2}\left(\hat{v}_{H}^{i}, \hat{E}_{y} ; \frac{\partial h_{T E}(\sigma)}{\partial \sigma_{j}}\right)=\left\langle\hat{v}_{H}^{i}, \hat{E}_{y}\right\rangle_{L^{2}(\Omega)} .
$$

A similar derivation for the TM mode can be found in the Appendix.

\section{Dimensionally Adaptive Inversion}

The dimensionality analysis of MT data is an ongoing and prevalent matter of study. Different works provide a criteria to discern when the MT data corresponds to a $1 \mathrm{D}, 2 \mathrm{D}$ or $3 \mathrm{D}$ problem. We highlight the contributions of Weaver et al. [40], who presented a dimensionality study based on the rotational invariants of the MT tensor, and the more recent work of Martí et al. [41], who based on these rotational invariants, implemented a software 
that provides a robust description of the dimensionality of the problem when dealing with real measurements.

In some scenarios, the dimension of the formation is unclear. Moreover, sometimes it is possible to interpret 3D MT data as 2D (see, for instance, [26] and [27]). Traditional inversion techniques usually select one fixed dimension (the full 2D (or 3D) problem) for both, the forward simulations and the inversion.

A proper and previous analysis of the media may recognize zones with different dimensionality signature. Therefore, it could be possible to identify some areas where the problem has higher dimensionality (2D or 3D), while others where a lower dimensionality (1D or 2D) may be sufficient. Based on this idea, we propose a Dimensionally Adaptive Method (DAM) for the inversion, in order to take advantage of this scenario.

Consistently, in this work we first solve a full 1D IP, with an already known (and fast) forward analytical solution that is employed to obtain explicitly the solution for the EM fields (see, e.g., Chapter 2 of [25]), and hence, the impedance. Once the 1D IP solution is obtained, we introduce it into the formulation of the 2D IP. That is:

- In the first step, we solve the IP for a 1D background model consisting of a layered media. From there, we obtain $\hat{m}_{1 D}$, the solution of the 1D IP. Notice that the corresponding forward solution is inexpensively computed analytically.

- After that, we consider the 1D solution obtained in the previous step as the initial point of the 2D IP. A complementary possibility is to define a regularization parameter of the form $R(m)=\left|m-\hat{m}_{1 D}\right|_{L^{p}(\Omega)}$, where $p$ is usually 1 or 2 . In our case, we do not use a regularization parameter in order to fairly compare the computational performance of the traditional vs. the DAM approach for the inversion.

- Finally, we solve the full 2D IP.

The main advantages of this approach are: (a) to be able to study separately $1 \mathrm{D}$ and $2 \mathrm{D}$ effects in the IP, (b) the $1 \mathrm{D}$ IP has less unknowns and is more stable, (c) employing this information in the $2 \mathrm{D}$ problem, we increase the robustness of the algorithm, (d) the total computational cost of performing the inversion is often lower. The extension of the DAM to 3D problems is straightforward. 


\section{Numerical Result}

\subsection{Model Problem}

Figure 1 describes the selected model problem for the full and secondary field formulations. The computational domain consists of air and a 1D layered media for modeling the subsurface formation with a 2D heterogeneity embedded in one of the layers. The horizontal dimension corresponds to the $x$ spatial variable (with zero at the center), and the vertical to $z$ (with zero on the surface). The size of the source is $(2500 \times 5) \mathrm{km}$ in the $x$ and $z$, and infinity in $y$. The forcing terms are $J_{y}^{i m p}=M_{y}^{i m p}=1$ and the physical domain is truncated with a PML complemented with a Dirichlet homogeneous $\mathrm{BC}$ imposed on its outer part.
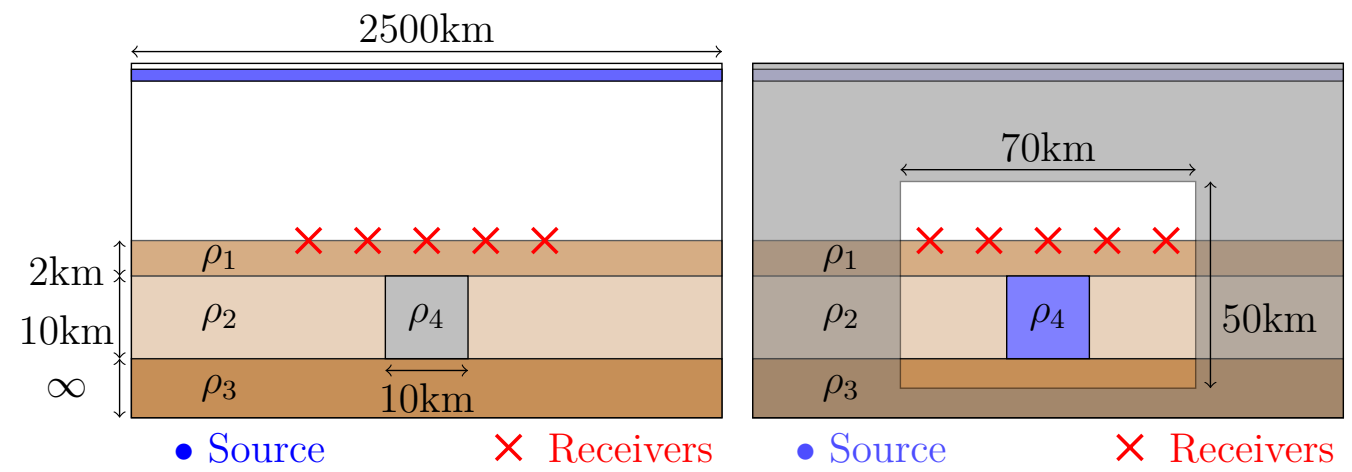
(a) 2D full field formulation.
(b) $2 \mathrm{D}$ secondary field formulation.

Figure 1: Model problems.

We consider two different models for the resistivity distribution of the Earth's subsurface (a conductive and a resistive anomaly), described in Table 1:

\begin{tabular}{ccccc}
\hline & $\rho_{1}$ & $\rho_{2}$ & $\rho_{3}$ & $\rho_{4}$ \\
\hline Model 1 & 80 & 100 & 120 & 10 \\
Model 2 & 3 & 2 & 4 & 200 \\
\hline
\end{tabular}

Table 1: Two different models for the subsurface described in Figure 1. 


\section{2. $h p$-Adaptivity}

We consider a source operating at $0.1 \mathrm{~Hz}$ and the resistivity distribution of the Model 1 and Table 1. Figure 2 displays the convergence history in terms of the $y$ component of the EM fields measured at the center of the domain, which is the region most influenced by the presence of the inhomogeneity in the considered model problem. In all cases, we observe the superior performance of the multi-goal oriented $h p$-adaptive algorithm. The initial slow convergence observed on the $h p$-adaptive algorithm for the TM mode is attributed to some pre-asymptotic convergence problems within the PML region. In fact, the convergence behavior is not necessarily monotone because we are considering the error in a quantity of interest rather than in a global norm.

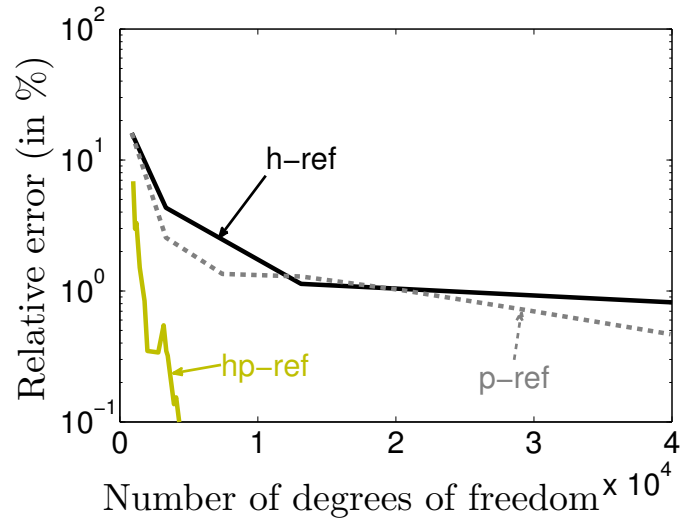

(a) TE mode.

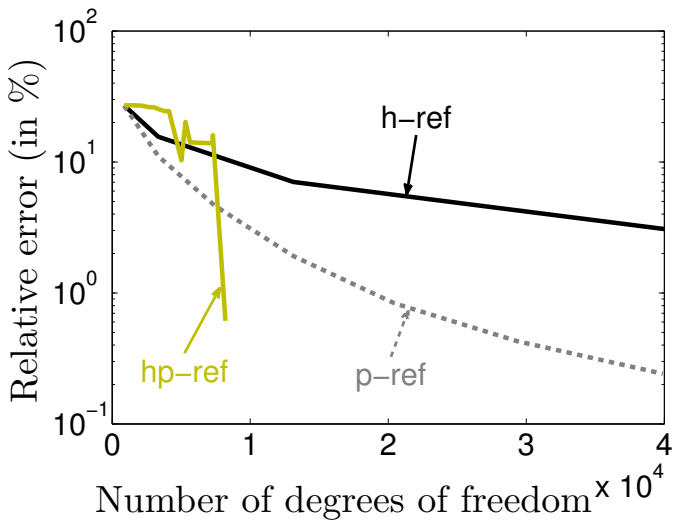

(b) TM mode.

Figure 2: Relative error in $E_{y}$ (TE mode) and $H_{y}$ (TM mode) vs. the number of degrees of freedom for three type of refinements: uniform $h$-refinements, uniform $p$-refinements and multi-goal oriented self-adaptive $h p$-refinements.

Figure 3 describes different $h p$ grids obtained after the adaptive process. We consider two different adaptive methods: the single goal and multi-goal oriented $h p$-adaptivity. For the former, the objective is to obtain accurate results at one receiver placed at the center of the domain, while the main goal for the latter is to procure accurate results at seven different receivers placed from -20 to $20 \mathrm{~km}$ in the x-direction and along the Earth's surface.

The multi-goal oriented algorithm produces additional refinements towards the sides of the domain, that is, towards the receivers, whereas the traditional goal-oriented algorithm focuses in the single receiver located at 
the center, as observed from Figure 3. This is specially notorious at the airground interface. Additionally, we observe that while in the TE mode it is also necessary to correctly refine in the air, for the TM mode the refinements are concentrated in the subsurface, as expected by the physical nature of the solution.

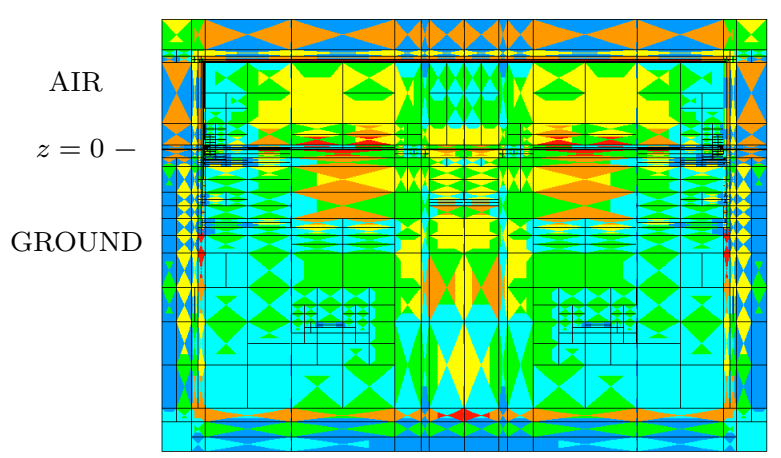

(a) Multi-goal oriented for TE mode.

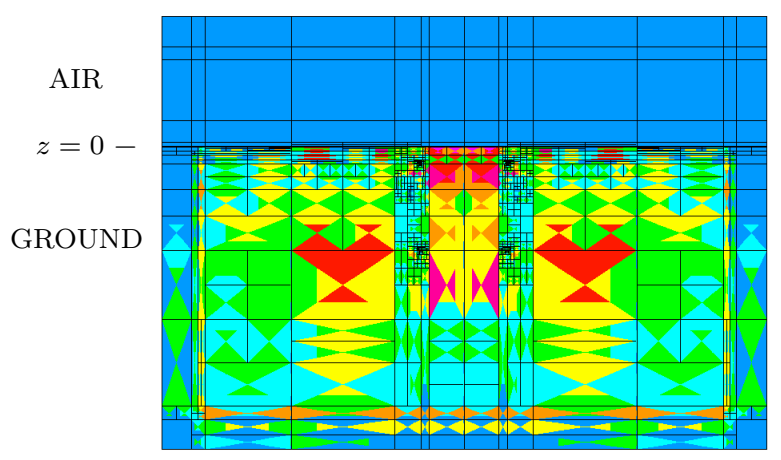

(c) Multi-goal oriented for TM mode.

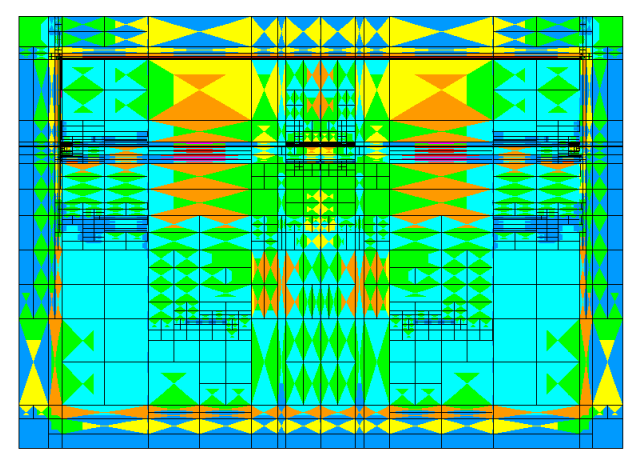

(b) Goal-oriented for TE mode.

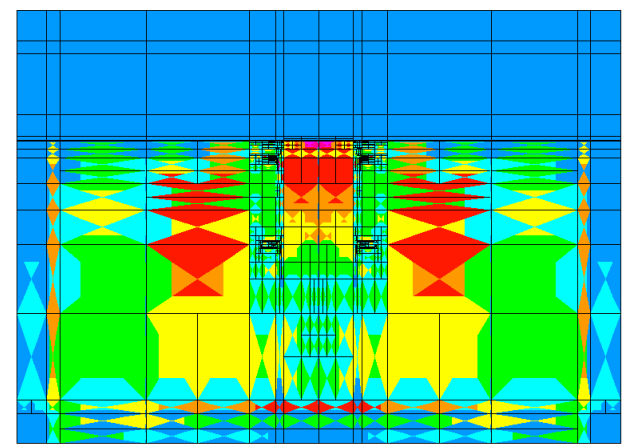

(d) Goal oriented for TM mode.

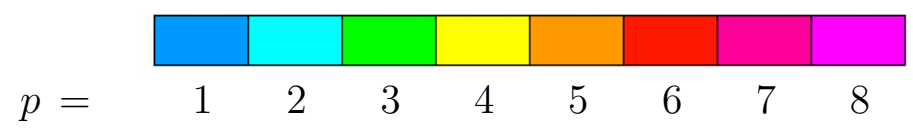

Figure 3: Different $h p$-grids after performing the adaptive algorithm for the TE and TM formulations with a frequency equal to $0.1 \mathrm{~Hz}$.

A sequence of $h p$-meshes are depicted in Figure 4 . The final grid contains 9765 unknowns and it delivers a relative error in $L\left(\hat{E}_{y}\right)$ below $1.17 \cdot 10^{-3} \%$. 


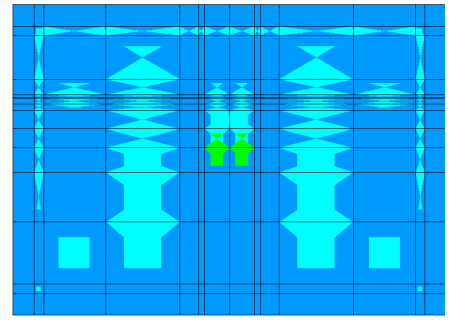

(a) Iteration 3 .

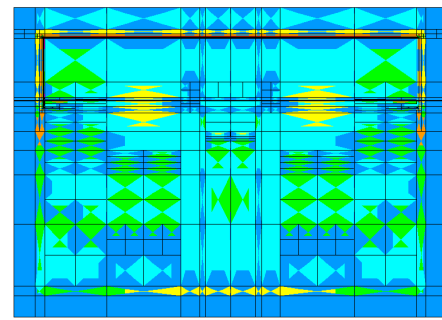

(d) Iteration 12.

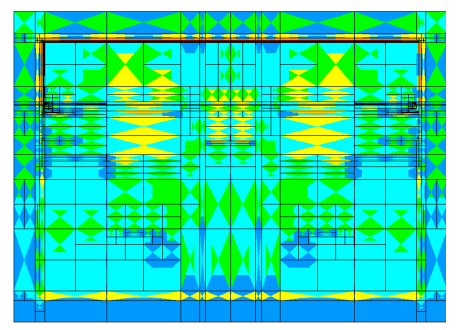

(g) Iteration 21.

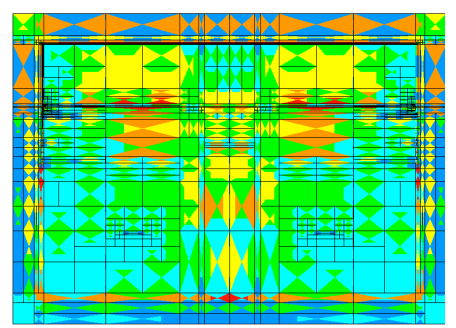

(j) Iteration 30 .

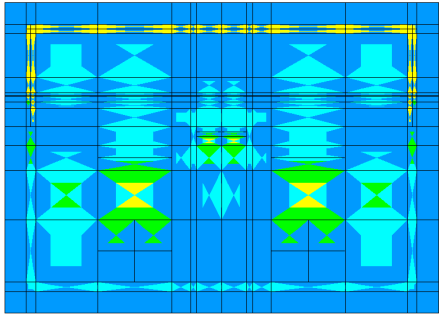

(b) Iteration 6.

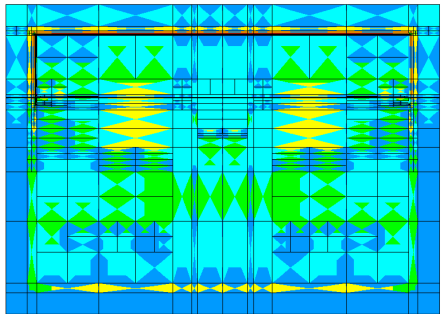

(e) Iteration 15.

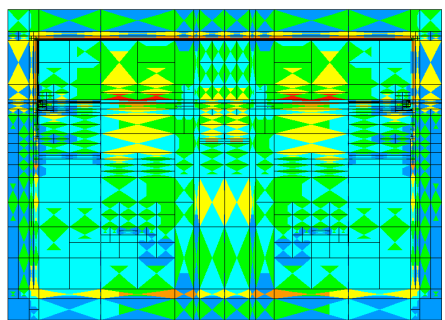

(h) Iteration 24.

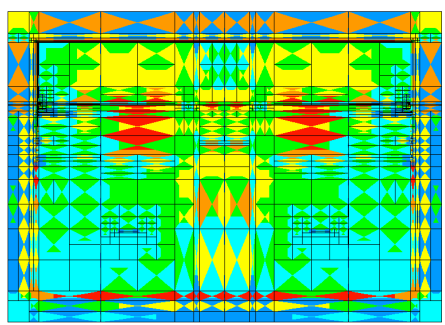

(k) Iteration 33.

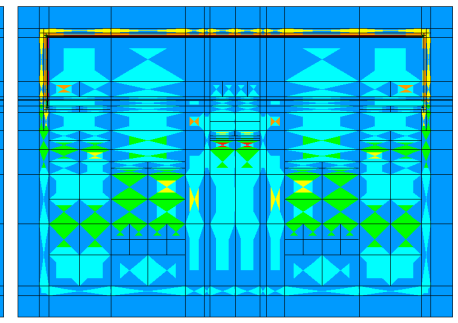

(c) Iteration 9 .

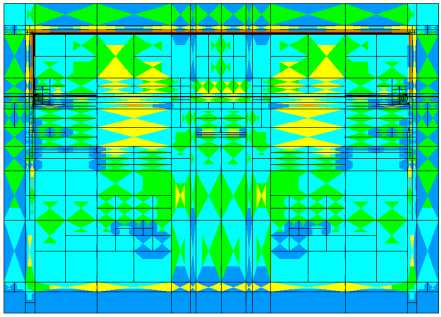

(f) Iteration 18.

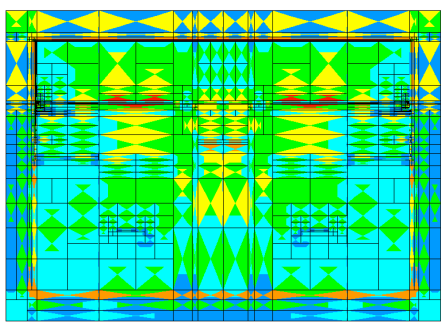

(i) Iteration 27.

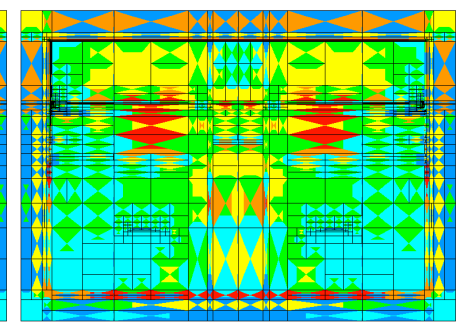

(1) Iteration 36.

Figure 4: Different $h p$-grids delivered by of the multi-goal oriented adaptive algorithm for the TE mode when the secondary field formulation is employed at $0.1 \mathrm{~Hz}$. 


\subsection{Full Field versus Secondary Field Formulation}

We now solve a 2D subsurface formation for the full and secondary field formulation problems. We consider an underlying 1D layered media for the primary field, and the same multi-goal oriented $h p$-FEM with a reduced domain to solve secondary field variations [23], as described in Model 1 and Table 1, operating at a frequency equal to $0.1 \mathrm{~Hz}$.

To estimate the relative errors, we employ an overkill solution with a sufficiently refined mesh that guarantees high accuracy. Figure 5 shows that the secondary field formulation requires less unknowns than the full field formulation in order to produce a comparable error level, as expected. Additionally, $p$-uniform refinements provide faster convergence when compared to $h$-uniform refinements. This difference between the performance of $p$ refinements versus $h$-refinements is more notorious in the TE mode than in the TM mode for the considered example.

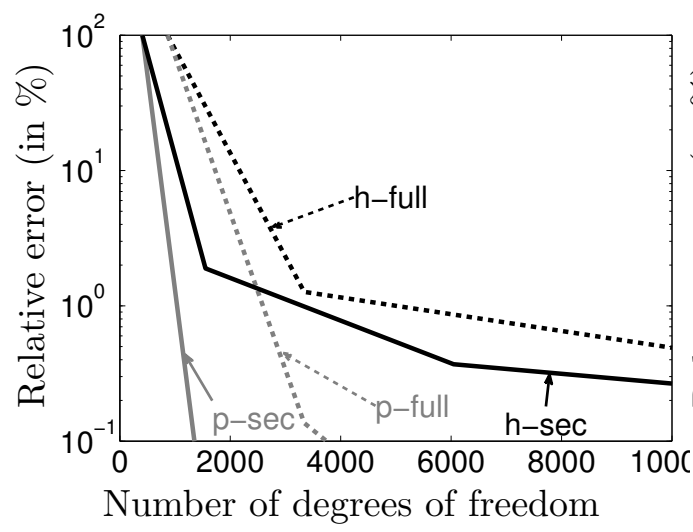

(a) TE mode.

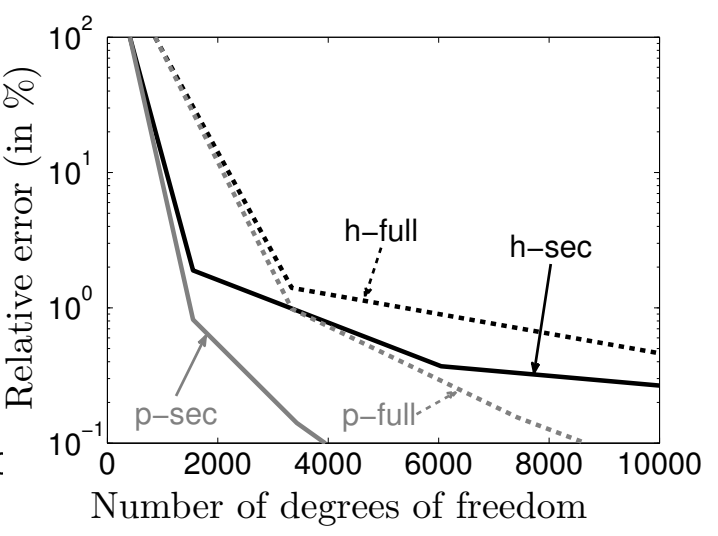

(b) TM mode.

Figure 5: Relative error in terms of the apparent resistivity computed with the full and secondary field formulations.

\subsection{Traditional Inverse Problem}

We consider only synthetic problems. To simulate the measurements, we use a highly refined mesh as follows: given a distribution of the subsurface $\hat{\rho}=\left\{\rho_{i}\right\}_{i}^{M}$, where $\hat{\rho}$ denotes the solution of the IP, we solve the forward direct problems and we define $d_{\omega_{j}}^{i, k}=\mathcal{Z}_{\omega_{j}}^{i, k}(\hat{\rho})$. We intentionally consider a different solver to simulate the measurements, in our case, the one associated to the secondary field formulation. To mimic realistic measurements, we add a $3 \%$ 
uncorrelated random Gaussian noise to the real and imaginary parts of the simulated measurements. Except for the simulation of the synthetic measurements, we employ the full field formulation to solve the forward problem in the rest of the inversion results. To solve the IP, we employ the L-BFGS-U method and we compute the derivatives of the impedance with respect the materials with equation (15).

\subsubsection{Selection of the Inversion Variables}

In this subsection, we analyze how different unknowns in the MT IP affect to the convergence of the optimization method. We consider three different variables, namely $m=\sigma$, its $\operatorname{logarithm} m=\log (\sigma)$, and $m=\rho=1 / \sigma$, and the cost functional given by equation (13), that is, without regularization parameter. Then, given an arbitrary initial point $\rho^{(0)}$ (described in Table 2), we are interested in recovering the true one $\hat{\rho}$.

\begin{tabular}{cc|c}
\hline$\rho^{(0)}$ & $\hat{\rho}$ \\
\hline Model 1 & $(40,40,40,40)$ & $(80,100,120,10)$ \\
Model 2 & $(25,25,25,25)$ & $(3,2,4,200)$ \\
\hline
\end{tabular}

Table 2: Initial point and solution for the considered IPs.

Seven receivers are located on the surface at different $x$-positions. They record measurements at the following four different frequencies in Hz: $\nu_{1}=$ $10^{-3}, \nu_{2}=10^{-2}, \nu_{3}=10^{-1}$, and $\nu_{4}=1$. Figures 6 displays the value of the cost functional against the iteration number of the L-BFGS-U optimization method, while Table 3 provides the solution to the IPs at the end of the iterative processes.

From these results, we conclude:

- The selected inversion unknown strongly affects to the convergence of the method. All inversion unknowns perform similarly for the TE mode, while for the TM mode, we are unable to solve the IP when employing the conductivity as the variable for the inversion. In Model 2 , the conductivity is the worst unknown for the inversion.. Since each iteration demands to solve $N_{\omega}$ forward direct problems and $N_{\omega} \times N_{\text {rec }}$ forward adjoint problems, a good selection of the inversion variable becomes important to reduce the computational cost of the inversion. For the considered examples, $m=\log (\sigma)$ seems the best option as an unknown, while $m=\sigma$, is always the worst (except in one case). 


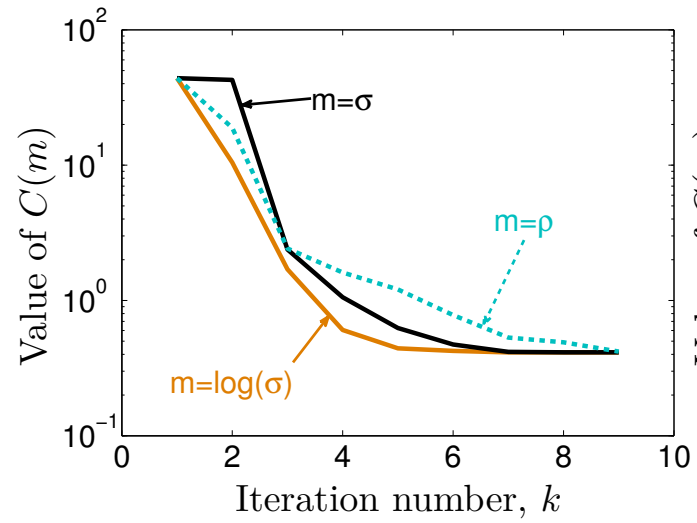

(a) TE mode.

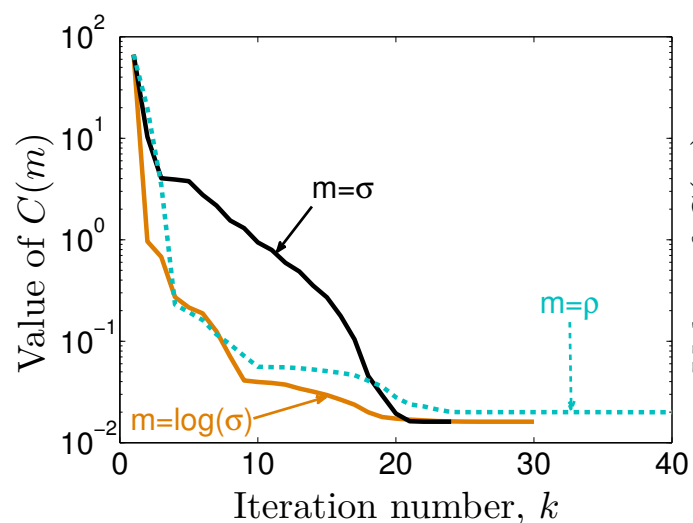

(c) TE mode.

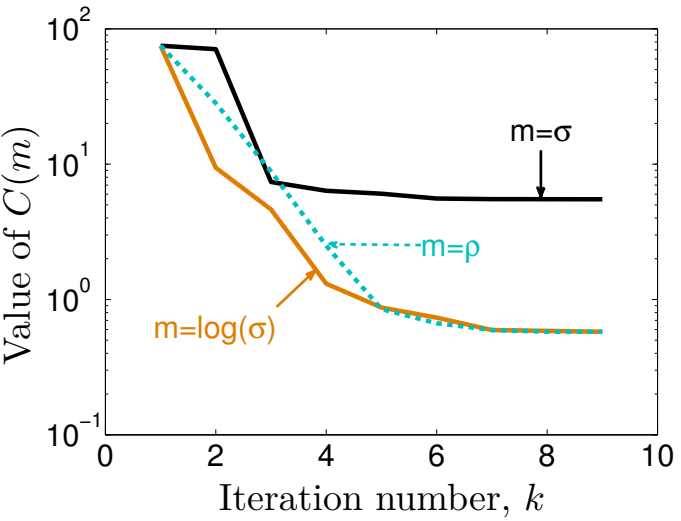

(b) TM mode.

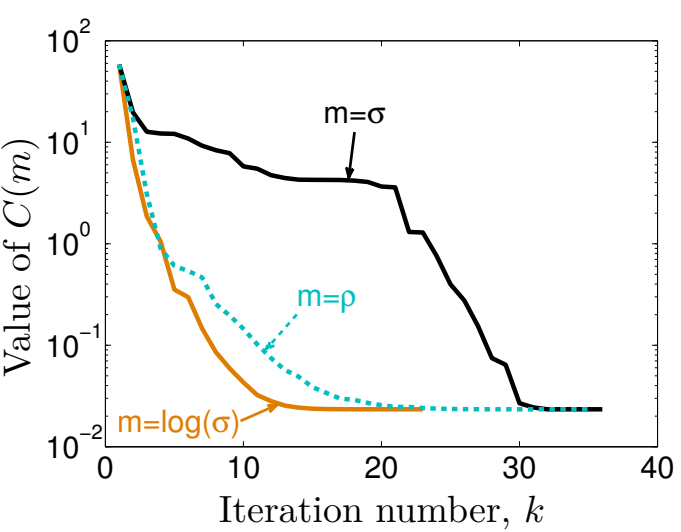

(d) TM mode.

Figure 6: Cost functional against the iteration number for Model 1 (above) and Model 2 (below) described in Table 1. 


\begin{tabular}{c|c|c||c||c|c|c} 
& \multicolumn{2}{|c|}{ Model 1 } & Solution & \multicolumn{2}{c|}{ Model 2 } & Solution \\
\hline$m=\sigma$ & TE & TM & - & TE & TM & - \\
\hline \hline$\rho_{1}$ & 84.30 & 77.34 & 80 & 3.01 & 2.99 & 3 \\
$\rho_{2}$ & 87.76 & 112.09 & 100 & 2.02 & 2.08 & 2 \\
$\rho_{3}$ & 119.66 & 118.02 & 120 & 4.02 & 3.74 & 4 \\
$\rho_{4}$ & 9.93 & 0.69 & 10 & 1000 & 432.94 & 200 \\
\hline \hline$m=\rho$ & - & - & - & - & - & - \\
\hline \hline$\rho_{1}$ & 84.72 & 81.48 & 80 & 3.02 & 2.99 & 3 \\
$\rho_{2}$ & 89.89 & 96.50 & 100 & 2.02 & 2.08 & 2 \\
$\rho_{3}$ & 120.16 & 124.42 & 120 & 4.03 & 3.74 & 4 \\
$\rho_{4}$ & 9.94 & 9.68 & 10 & 1000 & 426.50 & 200 \\
\hline \hline$m=l o g(\sigma)$ & - & - & - & - & - & - \\
\hline \hline$\rho_{1}$ & 83.73 & 81.25 & 80 & 3.02 & 2.99 & 3 \\
$\rho_{2}$ & 88.76 & 96.88 & 100 & 2.02 & 2.08 & 2 \\
$\rho_{3}$ & 119.81 & 124.17 & 120 & 4.02 & 3.74 & 4 \\
$\rho_{4}$ & 9.88 & 9.68 & 10 & 1000 & 426.07 & 200 \\
\hline
\end{tabular}

Table 3: Values of the resistivities for the solution of the inversion problem. 
- There is not a significant difference between the performance of the TE and TM modes.

- The most resistive layer of Model 2 is not accurately recovered, in part due to the effect of noise. Additionally, we have numerically observed that the value of the cost functional close to the solution does not change significantly when the value of $\rho_{4}$ varies between 150 and 1000 Ohm-m. This suggest that the cost functional presents a valley, and hence, it is barely sensitive to this parameter.

\subsection{2. $T E+T M$ Inversion}

We now consider the joint TE+TM inversion. Again, we solve the IP without regularization parameter in order to compare the results with the single mode inversion of the previous section. We consider the following four different frequencies in Hz: $\nu_{1}=10^{-3}, \nu_{2}=10^{-2}, \nu_{3}=10^{-1}$, and $\nu_{4}=1$. We place seven receivers, on the surface at different $x$-positions.

Figure 7a shows a comparison between the TE inversion, TM inversion and TE+TM inversion for Model 1 when the unknown for the inversion corresponds to the logarithm of the conductivity. The convergence curve is almost identical in the three cases, and the solution for the TE+TM inversion is $\hat{\rho}=(79.63,97.21,122.49,10.01)$, which is significantly more accurate than that obtained with any of the two single mode inversion. However, as counterpart, the computational cost of performing one iteration for the TE+TM inversion is doubled that corresponding to a single mode inversion.

Figure $7 \mathrm{~b}$ compares the performance of the same three modes when the resistivity is the unknown of the IP corresponding to the Model 2 and Table 2. While we are unable to accurately solve the IP for the TE mode nor the TM mode when considering them independently (see Table 3), the IP for the joint TE+TM inversion converges to a more accurate solution than the single modes solutions, namely, to $\hat{\rho}=(3.01,2.02,4.04,133.33)$.

\subsection{Dimensionally Adaptive Inversion}

We now compare the traditional approach (employed in the previous subsection) with the DAM approach. We consider the model problem depicted in Figure 1. The employed four different frequencies in $\mathrm{Hz}$ are $\nu_{1}=10^{-3}$, $\nu_{2}=10^{-2}, \nu_{3}=10^{-1}$, and $\nu_{4}=1$.

Lets denote by $\rho_{1 D}^{(0)}=\left(\rho_{1}^{(0)}, \rho_{2}^{(0)}, \rho_{3}^{(0)}\right)$ and $\hat{\rho}_{1 D}=\left(\hat{\rho}_{1}, \hat{\rho}_{2}, \hat{\rho}_{3}\right)$ to the initial point and the solution of the 1D IP, respectively, and lets denote by $\rho_{\text {trad }}^{(0)}$ and 


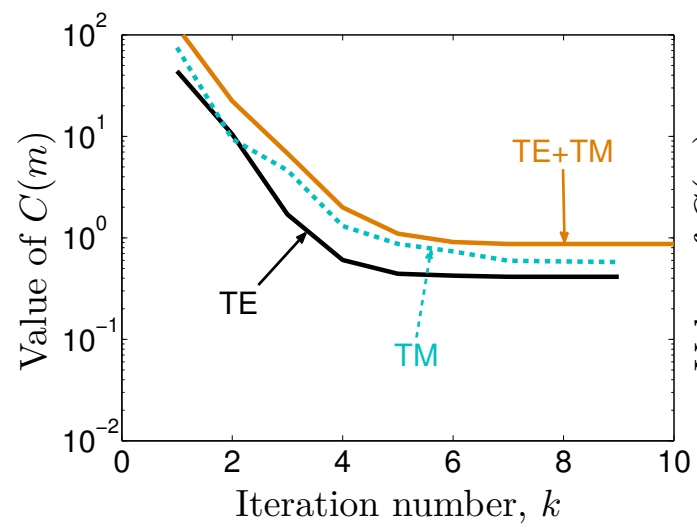

(a) Model 1, $m=\log (\sigma)$.

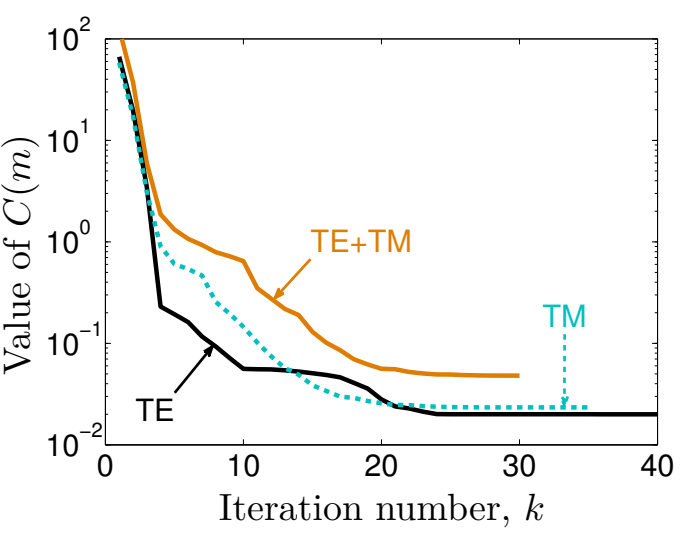

(b) Model 2, $m=\rho$.

Figure 7: Cost functional against the iteration number when different modes are employed in the inversion.

$\rho_{D A M}^{(0)}$ to the initial points for the 2D IP when the traditional and the DAM approaches are employed, respectively. We consider two different scenarios to compare the traditional and DAM approaches: the first one employs the TE mode and the conductivity as inversion variable, while the second one uses the joint TE+TM inversion and the resistivity as inversion variable. Table 4 summarizes the information of each of them.

\begin{tabular}{l|c|c|c|c}
\hline & $\rho_{1 D}^{(0)}$ & $\hat{\rho}_{1 D}$ & $\rho_{\text {trad }}^{(0)}$ & $\rho_{D A M}^{(0)}$ \\
\hline sc. 1 & $(25,25,25)$ & $(3.11,3.03,3.07)$ & $(25,25,25,25)$ & $(3.11,3.03,3.07,25)$ \\
sc. 2 & $(25,25,25)$ & $(3.06,4.95,4.92)$ & $(25,25,25,25)$ & $(3.06,4.95,4.92,25)$ \\
\hline
\end{tabular}

Table 4: Information of the two scenarios considered to compare the traditional and the DAM approaches for the inversion.

We show in Figure 8 that in addition to observe two orders of magnitude difference on the value of $C(m)$ at the beginning of the IP, thanks to the inclusion of physical relevant information contained on the underlying 1D problem, the algorithm employing the DAM approach converges significantly faster. For this particular scenarios, it only requires approximately $25 \%$ of the iterations, which implies significant computational savings. 

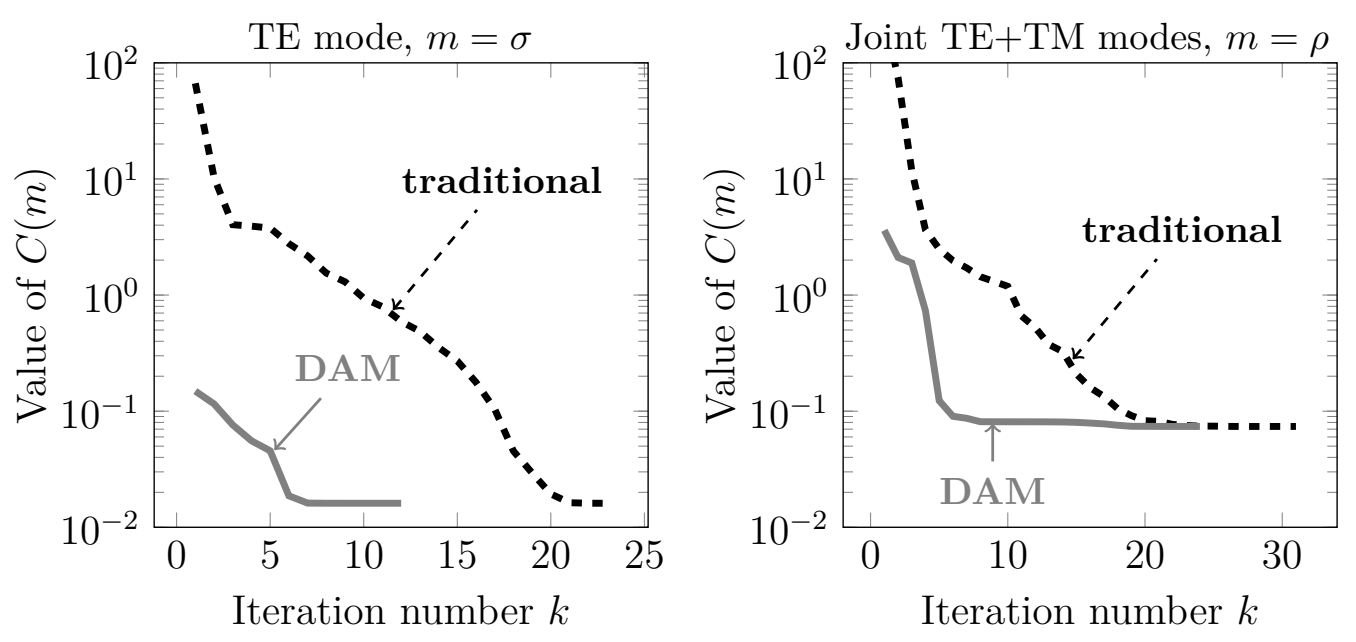

Figure 8: Cost functional against the iteration number for the traditional and DAM IPs for Model 2 and Table 1.

\section{Conclusions}

We have introduced a multi-goal oriented $h p$-adaptive FEM formulation for simulating and inverting MT problems. In particular, we have illustrated the fast convergence of the multi-goal oriented self-adaptive $h p$-FEM in terms of the error vs. the problem size, as expected from the theory [16, 17], we have shown a sequence of optimal $h p$-grids in the process of constructing a final grid with the multi-goal oriented adaptive algorithm, and we have highlighted that the secondary field formulation requires less unknowns than the full field formulation in order to produce a comparable error level in context of hp-FEM simulations.

The Dimensionality Adaptive Method (DAM) has shown its capability to reduce the computational cost associated to the IP when prior knowledge of the dimensionality is available. In our particular problem where a 1D layered underlying media is considered, the inclusion of physical relevant information related to the $1 \mathrm{D}$ IP solution into the $2 \mathrm{D}$ IP reduces the total number of iterations by $75 \%$. Notice that when employing a regularization parameter, this approach is expected to be more robust.

Additionally, we have analyzed how the choice of the optimal inversion variable should be problem dependent, and we have seen that the joint TE + TM inversion provides more accurate results for the IP than single modes inversion. Finally, we have also provided a formulation for implementing an 
efficient adjoint based method for the computation of the derivatives of the impedance (a nonlinear QoI) in terms of linear QoI.

\section{Appendix A Jacobian for the TM Mode}

To compute the entries of $\left[\mathcal{J}_{L_{\sigma}}\right]_{j}^{i}$ for the TM mode, we define the following linear operator:

$$
L_{T M}^{i}(u)=\frac{1}{(\sigma+j \omega \varepsilon)\left|\Omega_{R^{i}}\right|} \int_{\Omega_{R^{i}}} \frac{\partial u}{\partial z} d \Omega .
$$

$L_{T M}^{i}(u)$ is defined according to the equation for $E_{x}$ given by Maxwell's equations. This EM field component is needed for computing the impedance.

- If $P(u)=L^{i}(u)$ :

$$
\begin{array}{ll}
D: & b(v, \hat{u})=G(v), \quad \forall v \in V, \\
A: & b\left(\hat{v}_{H}^{i}, u\right)=L^{i}(u), \quad \forall u \in V .
\end{array}
$$

From (28), we obtain the solution for $\hat{H}_{y}=\hat{u}$, and we compute the associated linear QoI as $L^{i}\left(\hat{H}_{y}\right)$. By solving (29), we obtain the solution for $\hat{v}_{H}^{i}$. Since (28) holds for all $v$, in particular it is also valid for $\hat{v}_{H}^{i}$. Using equation (17) we have that:

$$
\frac{\partial L^{i}\left(\hat{H}_{y}\right)}{\partial \sigma_{j}}=-b_{2}\left(\hat{v}_{H}^{i}, \hat{H}_{y} ; \frac{\partial h_{T M}(\sigma)}{\partial \sigma_{j}}\right)=-\left\langle\nabla \hat{v}_{H}^{i}, \frac{1}{\left(\sigma_{j}+j \omega \varepsilon\right)^{2}} \nabla \hat{H}_{y}\right\rangle_{L^{2}(\Omega)} .
$$

- If $P(u)=L_{T M}^{i}(u)$ :

$$
\begin{array}{ll}
D: & b(v, \hat{u})=G(v), \quad \forall v \in V, \\
A: & b\left(\hat{v}_{E}^{i}, u\right)=L_{T M}^{i}(u), \quad \forall u \in V,
\end{array}
$$

Since $\hat{H}_{y}$ is already computed, from Maxwell's equations we have that

$$
L^{i}\left(\hat{E}_{x}\right)=L_{T M}^{i}\left(\hat{H}_{y}\right) .
$$

Following the same argument, solving equation (32) we obtain the solution for $\hat{v}_{E}^{i}$, and using the Representation Theorem stated above, we obtain that:

$$
\begin{aligned}
\frac{\partial L^{i}\left(\hat{E}_{x}\right)}{\partial \sigma_{j}} & =\frac{\partial L_{T M}^{i}\left(\hat{H}_{y}\right)}{\partial \sigma_{j}}=-b_{2}\left(\hat{v}_{E}^{i}, \hat{H}_{y} ; \frac{\partial h_{T M}(\sigma)}{\partial \sigma_{j}}\right)+\frac{\partial h_{T M}(\sigma)}{\partial \sigma_{j}} \int_{\Omega_{R^{i}}} \frac{\partial \hat{H}_{y}}{\partial z} d \Omega \\
& =-\left\langle\nabla \hat{v}_{E}^{i}, \frac{1}{\left(\sigma_{j}+j \omega \varepsilon\right)^{2}} \nabla \hat{H}_{y}\right\rangle_{L^{2}(\Omega)}-\frac{1}{\left(\sigma_{j}+j \omega \varepsilon\right)^{2}} \int_{\Omega_{R^{i}}} \frac{\partial \hat{H}_{y}}{\partial z} d \Omega
\end{aligned}
$$


Notice that now $L_{T M}^{i}\left(\hat{H}_{y}\right)$ depends on $\sigma$ and therefore we have to add a new term that is zero for the domains where $\Omega_{R^{i}} \cap \Omega_{\sigma_{j}}=\emptyset$.

\section{Acknowledgments}

Julen Alvarez-Aramberri and David Pardo were partially funded by the Project of the Spanish Ministry of Economy and Competitiveness with reference MTM2013-40824-P, the BCAM "Severo Ochoa" accreditation of excellence SEV-2013-0323, and the Basque Government through the BERC 2014-2017 program and the Consolidated Research Group Grant IT649-13 on "Mathematical Modeling, Simulation, and Industrial Applications (M2SI)". David Pardo has received funding from the European Union's Horizon 2020 research and innovation programme under the Marie Sklodowska-Curie grant agreement No 644602, by the RISE Horizon 2020 European Project GEAGAM (644602).

\section{References}

[1] A. Tikhonov, On determining electrical characteristics of the deep layers of the Earth's crust, in: Sov. Math. Dokl, Vol. 2, 1950, pp. 295-297.

[2] L. Cagniard, Basic theory of the magneto-telluric method of geophysical prospecting, Geophysics 18 (3) (1953) 605-635.

[3] X. Ogaya, J. Ledo, P. Queralt, A. Marcuello, A. Quintà, First geoelectrical image of the subsurface of the Hontomín site (Spain) for $\mathrm{CO}_{2}$ geological storage: A magnetotelluric 2D characterization, International Journal of Greenhouse Gas Control 13 (2013) 168-179.

[4] M. Unsworth, A. Jones, W. Wei, G. Marquis, S. Gokarn, J. Spratt, P. Bedrosian, J. Booker, C. Leshou, G. Clarke, et al., Crustal rheology of the Himalaya and Southern Tibet inferred from magnetotelluric data, Nature 438 (7064) (2005) 78-81.

[5] P. Kantek, P. Balbi, F. Ramos, H. Fraga de Campos, Electromagnetic induction in three-dimensional structures, in: Inverse Problems in Engineering: Theory and Practice 3rd Int. Conference on Inverse Problems in Engineering, Port Ludlow, WA, USA, 1999. 
[6] Y. Ogawa, M. Mishina, T. Goto, H. Satoh, N. Oshiman, T. Kasaya, Y. Takahashi, T. Nishitani, S. Sakanaka, M. Uyeshima, et al., Magnetotelluric imaging of fluids in intraplate earthquake zones, ne japan back arc, Geophys. Res. Lett 28 (19) (2001) 3741-3744.

[7] R. Hyndman, P. Shearer, Water in the lower continental crust: modelling magnetotelluric and seismic reflection results, Geophysical Journal International 98 (2) (1989) 343-365.

[8] T. Hughes, J. Cottrell, Y. Bazilevs, Isogeometric analysis: CAD, finite elements, NURBS, exact geometry and mesh refinement, Computer methods in applied mechanics and engineering 194 (39) (2005) 41354195.

[9] D. Schillinger, S. Hossain, T. Hughes, Reduced Bézier element quadrature rules for quadratic and cubic splines in isogeometric analysis, Computer Methods in Applied Mechanics and Engineering 277 (2014) 1-45.

[10] L. Demkowicz, J. Gopalakrishnan, A class of discontinuous PetrovGalerkin methods. Part I: the transport equation, Computer Methods in Applied Mechanics and Engineering 199 (23) (2010) 1558-1572.

[11] J. Zitelli, I. Muga, L. Demkowicz, J. Gopalakrishnan, D. Pardo, V. Calo, A class of discontinuous Petrov-Galerkin methods. part IV: the optimal test norm and time-harmonic wave propagation in 1D, Journal of Computational Physics 230 (7) (2011) 2406-2432.

[12] L. Demkowicz, Computing with $h p$-Adaptive Finite Elements: volume 1. One and Two Dimensional Elliptic and Maxwell problems, CRC Press, 2006.

[13] W. Rachowicz, D. Pardo, L. Demkowicz, Fully automatic $h p$-adaptivity in three dimensions, Computer Methods in Applied Mechanics and Engineering 195 (37) (2006) 4816-4842.

[14] L. Demkowicz, P. Gatto, J. Kurtz, M. Paszyński, W. Rachowicz, E. Bleszyński, M. Bleszyński, M. Hamilton, C. Champlin, D. Pardo, Modeling of bone conduction of sound in the human head using $h p$-finite elements: Code design and verification, Computer Methods in Applied Mechanics and Engineering 200 (21) (2011) 1757-1773. 
[15] I. Gomez-Revuelto, L. Garcia-Castillo, S. Llorente-Romano, D. Pardo, 3D $h p$-adaptive finite element simulations of bend, step, and magic-T electromagnetic waveguide structures, Journal of Computational Science 5 (2) (2014) $65-75$.

[16] W. Gui, I. Babuska, The $h, p$ and $h p$ versions of the finite element method in 1 dimension. I-III, Numerische Mathematik 49 (6) (1986) 577-683.

[17] I. Babuška, B. Guo, Approximation properties of the $h-p$ version of the finite element method, Computer Methods in Applied Mechanics and Engineering 133 (3) (1996) 319-346.

[18] R. Becker, R. Rannacher, Weighted a posteriori error control in FE methods, IWR, 1996.

[19] D. Pardo, Multigoal-oriented adaptivity for $h p$-finite element methods, Procedia Computer Science 1 (1) (2010) 1953-1961.

[20] J. Alvarez-Aramberri, D. Pardo, H. Barucq, Inversion of magnetotelluric measurements using multigoal oriented $h p$-adaptivity, Procedia Computer Science 18 (2013) 1564-1573.

[21] J. Alvarez-Aramberri, D. Pardo, H. Barucq, Automatically adapted perfectly matched layers for problems with high contrast materials properties, Procedia Computer Science 29 (2014) 970-979.

[22] J. Berenger, A perfectly matched layer for the absorption of electromagnetic waves, Journal of computational physics 114 (2) (1994) 185-200.

[23] J. Alvarez-Aramberri, D. Pardo, H. Barucq, A secondary field based $h p$-finite element method for the simulation of magnetotelluric measurements, Journal of Computational Science 11 (2015) 137-144.

[24] J. Alvarez-Aramberri, S. Bakr, D. Pardo, H. Barucq, Quantities of interest for surface based resistivity geophysical measurements, Procedia Computer Science 51 (2015) 964-973.

[25] W. Chew, Waves and fields in inhomogeneous media, Vol. 522, IEEE press New York, 1995. 
[26] J. Ledo, P. Queralt, A. Martí, A. Jones, Two-dimensional interpretation of three-dimensional magnetotelluric data: an example of limitations and resolution, Geophysical Journal International 150 (1) (2002) 127139 .

[27] J. Ledo, 2-d versus 3-d magnetotelluric data interpretation, Surveys in Geophysics 26 (5) (2005) 511-543.

[28] A. Medin, The magnetotelluric inverse problem, Ph.D. thesis, University of California, San Diego (2008).

[29] R. Byrd, P. Lu, J. Nocedal, C. Zhu, A limited memory algorithm for bound constrained optimization, SIAM Journal on Scientific Computing 16 (5) (1995) 1190-1208.

[30] J. Alvarez-Aramberri, $h p$-adaptive simulation and inversion of magnetotelluric measurements, Ph.D. thesis, University of Basque Country and University of Pau in MAGIQUE 3D Group (INRIA) (2015).

[31] D. Pardo, L. Demkowicz, C. Torres-Verdín, L. Tabarovsky, A goaloriented $h p$-adaptive finite element method with electromagnetic applications. Part I: electrostatics, International Journal for Numerical Methods in Engineering 65 (8) (2006) 1269-1309.

[32] D. Pardo, L. Demkowicz, C. Torres-Verdin, M. Paszynski, A selfadaptive goal-oriented $h p$-finite element method with electromagnetic applications. Part II: electrodynamics, Computer methods in Applied Mechanics and Engineering 196 (37) (2007) 3585-3597.

[33] MUMPS, A multifrontal massively parallel sparse direct solver, http://graal.ens-lyon.fr/MUMPS/ (2015).

[34] I. Gomez-Revuelto, L. Garcia-Castillo, L. Demkowicz, A comparison between PML, infinite elements and an iterative BEM as mesh truncation methods for $h p$ self-adaptive procedures in electromagnetics, Progress In Electromagnetics Research 126 (2012) 499-519.

[35] C. Zhu, R. Byrd, P. Lu, J. Nocedal, Algorithm 778: L-BFGS-B: Fortran subroutines for large-scale bound-constrained optimization, ACM Transactions on Mathematical Software (TOMS) 23 (4) (1997) 550-560. 
[36] D. Avdeev, A. Avdeeva, 3D magnetotelluric inversion using a limitedmemory quasi-Newton optimization, Geophysics 74 (3) (2009) F45-F57.

[37] J. Nocedal, Updating quasi-Newton matrices with limited storage, Mathematics of computation 35 (151) (1980) 773-782.

[38] R. Byrd, J. Nocedal, R. Schnabel, Representations of quasi-Newton matrices and their use in limited memory methods, Mathematical Programming 63 (1) (1994) 129-156.

[39] S. Prudhomme, J. Oden, On goal-oriented error estimation for elliptic problems: application to the control of pointwise errors, Computer Methods in Applied Mechanics and Engineering 176 (1) (1999) 313-331.

[40] J. Weaver, A. Agarwal, F. Lilley, Characterization of the magnetotelluric tensor in terms of its invariants, Geophysical Journal International 141 (2) (2000) 321-336.

[41] A. Martí, P. Queralt, J. Ledo, Waldim: A code for the dimensionality analysis of magnetotelluric data using the rotational invariants of the magnetotelluric tensor, Computers \& Geosciences 35 (12) (2009) 22952303. 